\title{
L-Arginine Protects from Pringle Manoeuvere of Ischemia-Reperfusion Induced Liver Injury
}

\author{
Pronobesh Chattopadhyay, ${ }^{*, a, b}$ Navneet Verma, ${ }^{a}$ Anurag Verma, ${ }^{a}$ Tirath Kamboj, ${ }^{a}$ \\ Nazam Ali KHAN, ${ }^{a}$ and Arun Kumar WAHI ${ }^{a}$ \\ ${ }^{a}$ College of Pharmacy IFTM; Lodhipur RajputMoradabad-244001U.P., India: and ${ }^{b}$ Birla Institute of Technology and \\ Sciences; Pilani-333031 Rajasthan, India.
}

Received November 22, 2007; accepted February 6, 2008; published online February 12, 2008

Pringle described a new technique to reduce blood loss during liver surgery. Adult Wistar rats were subjected to $1 \mathrm{~h}$ of partial liver ischemia and followed by $3 \mathrm{~h}$ reperfusion. Eighteen Wistar rats were divided into sham-operated control group (I) $(n=6)$, ischemia and reperfusion (I/R) group (II) $(n=6)$, L-arginine treated group $(100 \mathrm{mg} / \mathrm{kg}$ body weight/daily by oral route for $7 \mathrm{~d}$ before induced ischemia reperfusion maneuver) (III) $(n=6)$. Ischemic and reperfusion hepatocellular injury occurred as indicated by increased-alanine transaminase (ALT), aspartate transaminase (AST). Pre-treatment with L-arginine significantly decreased serum-ALT, AST after $1 \mathrm{~h}$ ischemia followed by $3 \mathrm{~h}$ of reperfusion. Nitric oxide production, in hepatocytes was increased 2 fold and MDA levels significantly decreased by L-arginine treatment as compared to I/R rat. Histopathology and TEM studies showed markedly diminished hepatocellular injury in L-arginine pretreated rats during the hepatic $\mathbf{I} / \mathbf{R}$, which reached a level comparable to saline-treated rat of sham operated group. Thus, findings it may be concluded that L-arginine afforded significant protection from hepatobiliary function from $\mathrm{I} / \mathrm{R}$ injury by nitric oxide production.

Key words Pringle manoeuvere; ischemic reperfusion injury; nitric oxide; hepatocellular injury; L-arginine

During hepatic resection, the risk of severe intra-operative bleeding is a major risk. ${ }^{1)}$ To prevent massive blood loss, continuous or intermittent vascular clamping of the hepatic artery and portal vein ligation is known as "Pringle manoeuvere" is an efficient method to reduce hemorrhage. ${ }^{2,3)}$ "Pringle manoeuvere" is a technique which leads to ischemic and reperfusion injury. Ischemia and reperfusion results in complex metabolic, ${ }^{4)}$ immunological ${ }^{5)}$ and micro vascular $\left.{ }^{6}\right)$ changes, which together may contribute to hepatocellular damage and dysfunction. ${ }^{7)}$ Nitric oxide (NO) is an important modulator of tissue blood flow, arterial pressure, neurotransmission, and immune cell function. ${ }^{8)}$ There is evidence that implicates $\mathrm{NO}$ as a modulator of the adhesive interactions among leukocytes, platelets, and endothelial cells ${ }^{9)}$ and $\mathrm{L}-$ arginine at a dose level $100 \mathrm{mg} / \mathrm{kg} /$ p.o. daily showed significantly protect from alcoholic injury. ${ }^{10)}$ It has been shown that NO-donating compounds provide significant protection against the micro vascular dysfunction that is normally associated with ischemia and reperfusion $(\mathrm{I} / \mathrm{R}){ }^{10)} \mathrm{L}$-Arginine is the precursor of NO in vivo and confirmed sustained NO production via L-arginine administration ameliorated effects of intestinal I/R injury. ${ }^{11)}$ However the effect of L-arginine therapy in the view of NO mediated vasodilatation on hepatic ischemia has not yet been completely elucidated. Therefore, this study was designed to investigate how an altered bioavailability of hepatocytes NO produces vasodilatation in hepatic artery supplementation by L-arginine affects the sinusoidal reperfusion and apoptosis under defined surgical techniques i.e. portal triad clamping (Pringle manoeuvere).

\section{MATERIALS AND METHODS}

Chemicals Collagenase was purchased from Hymedia (Mumbai, India), peroxides-conjugated goat anti-rabbit secondary antibody was purchased from Bio source international (NY, U.S.A.). Alanine transaminase (ALT), aspartate transaminase (AST) kits were procured from Merck India Ltd. (India) and solvents of analytical grade were purchased from Merck (India).

Animals Treatment Eighteen Wistar rats were divided into sham-operated control group (I) $(n=6)$, ischemia and reperfusion group (II) were given $0.9 \%$ saline $(5 \mathrm{ml} / \mathrm{kg}$, p.o.) for $7 \mathrm{~d}(n=6)$, L-arginine pretreated group $(100 \mathrm{mg} / \mathrm{kg}$ body weight/daily by oral route for $7 \mathrm{~d}$ before induced ischemia reperfusion maneuver) (III) $(n=6)$. All rats were treated in accordance with the guideline for the Care and Use of Laboratory Animals (NIH Publication No. 86-23, revised 1985) with the permission of institute ethical committee. The hepatic I/R protocol were performed as described earlier. ${ }^{12)}$

Procurement of Tissue and Blood A portion of the ischemic and non-ischemic liver lobe was fixed in buffered $10 \%$ formalin and Karnovsky's solution for histopathology and transmission electron microscopy (TEM) studies. Blood samples were obtained from the right ventricle via a left anterior thoracotomy at the time of sacrifice. The blood from different groups rats were collected in sterile syringes without anticoagulant and centrifuged to separate the serum. The serum samples were stored at $-20^{\circ} \mathrm{C}$ until use for ALT, AST, assays.

Biochemical Studies Hepatocytes were prepared by the collagenase perfusion method as described by Berry and Friend $^{13)}$ and hepatocytes NO were measured as described by Hibbs et al. ${ }^{14)}$ Serum alanine transaminase (ALT) [EC 2.6.1.2] and aspartate transaminase (AST) [EC 2.6.1.1] estimated by Merck kits (Merck India Ltd., Mumbai, India) according to the manufacturer's instructions. Liver was homogenized with five volumes of $50 \mathrm{~mm}$ Tris- $\mathrm{HCl}$ buffer $(\mathrm{pH} 7.3)$ containing 0.25 (м) sucrose, $10 \mathrm{~mm} \beta$-mercaptoethanol, $1 \mathrm{~mm}$ phenyl methyl sulfonyl fluoride and 1 mm EDTA using Potter Elvehjem homogenizer and lipid peroxidation was determined by estimating malondialdehyde (MDA) as described by Jordan and Schenkman. ${ }^{15)}$ 
Morphological Evaluation by Light Microscope Assay Serial of slices of liver tissues were prepared from rat in each group and stained with hematoxylin-eosin (H\&E) and then observed the histological changes under light microscope at $200 \times$ or $400 \times$ magnification.

Transmission Electron Microscopy Assay Liver tissue were fixed in Karnovsky's solution $\mathrm{pH} 7.4$ for $4 \mathrm{~h}$ at $4^{\circ} \mathrm{C}$ and postfixed with glutaraldehyde and osmium trioxide respectively. Thick sections were cut and ultra thin sections (70 nm) were stained with uranyl acetate followed by lead citrate and viewed under Moragagni 268D electron microscope (Netherlands).

Statistical Analysis All values were expressed as mean \pm S.D. Differences in mean values were compared using SPSS 11.0 by one-way ANOVA and Student-Newman-Keul (SNK) test. $p<0.05$ was considered as statistically significant.

\section{RESULTS AND DISCUSSION}

The results of ALT, AST, NO and MDA are shown in Table 1 . After $1 \mathrm{~h}$ ischemia followed by $3 \mathrm{~h}$ reperfusion of liver, activities of AST, ALT and MDA were reduced $(p<0.05)$ by L-arginine pretreatment compared to I/R group. Production of NO in isolated hepatocytes increased significantly $(p<0.01)$ in L-arginine pretreated group as compared to $\mathrm{I} / \mathrm{R}$ group. Earlier studies, showed that apoptosis and necrotic cells initial peak was after $1 \mathrm{~h}$ ischemia and followed by $30 \mathrm{~min}$ reperfusion and second peak was after $1 \mathrm{~h}$ ischemia and $3 \mathrm{~h}$ reperfusion followed by gradual decline of necrosis and apoptosis, ${ }^{16}$ ) therefore, present, investigation was designed to study after $1 \mathrm{~h}$ ischemia followed by $3 \mathrm{~h}$ reperfusion. Liver histopathology was evaluated based on sinusoidal congestion, cytoplasmic vacuolization, hepatocellular necrosis, and neutrophil infiltration. Administration of $\mathrm{L}-$ arginine reduced hepatocellular injury in $\mathrm{I} / \mathrm{R}$ rats (Fig. 1C)

Table 1. Effect of L-Arginine on AST, ALT, MDA and NO Levels after Ischemia Reperfusion of Rat Liver

\begin{tabular}{lccc}
\hline \hline & Sham-operated & I/R injury rat & L-Arginine treated \\
\hline $\left.\mathrm{ALT}^{a}\right)$ & $84.72 \pm 8.2$ & $1507.36 \pm 30.58^{* *}$ & $187.21 \pm 14.52^{* \# \#}$ \\
$\mathrm{AST}^{a)}$ & $56.02 \pm 11.92^{*}$ & $817.40 \pm 14.52^{* *}$ & $105.10 \pm 8.92^{* * \# \#}$ \\
$\mathrm{NO}^{b}$ & $11.09 \pm 4.81$ & $8.20 \pm 0.57^{*}$ & $26.57 \pm 6.30^{* * \#}$ \\
$\mathrm{MDA}^{c)}$ & $159.67 \pm 5.24$ & $252.10 \pm 7.92^{* *}$ & $167.32 \pm 4.96^{* \# \#}$
\end{tabular}

Results are expressed as mean \pm S.D. $(n=6)$. Significantly different $(* p<0.05$ $* * p<0.01)$ from sham operated group. Significantly different $(\# p<0.05$, \# $p<0.01)$ from I/R injury group. a) Expressed in IU/1. b) Expressed in pmol $/ \mathrm{min} / \mathrm{mg}$ of protein. c) Expressed in $\mathrm{nmol} / \mathrm{g}$ of liver tissue. which correlates with decrease plasma ALT and AST levels (Table 1). In liver, I/R induced injury is characterized by reduction in the numbers of perfused sinusoids, heaptocellular hypoxia and leakage of enzymes (ALT, AST) from hepatocytes. ${ }^{17)}$ Ischemia activates kupffer cells, which are the main sources of vascular reactive oxygen formation during the initial reperfusion period. ${ }^{4)}$ This effect is only observed after no-flow ischemia (Pringle transplantation) but not after hemorrhagic shock, i.e., low-flow ischemia. ${ }^{18)} \mathrm{NO}$ is a potent vasodilator, which diffuses freely across cell membranes and acts as intracellularly by activation of guanylate cyclase. In response to vasoconstriction by Pringle manoeuvere technique, NO can induce vasodilatation at the level of the sinusoids as well as at presinusoidal sites. ${ }^{19)} \mathrm{L}$-Arginine is precursor of NO and our study shows that pretreatment with Larginine increased NO production in hepatocytes which lead to heapatic artery dilatation and protects against liver damage from $\mathrm{I} / \mathrm{R}$ injury which focused insight into the underlying mechanisms of the effects of L-arginine.

Reperfusion of the ischemic liver caused hepatocellular necrosis and sinusoidal congestion by $1 \mathrm{~h}$ ischemia followed by $3 \mathrm{~h}$ of reperfusion in saline-treated I/R rats (Fig. 1B). There was severe sparing of the periportal areas with progressive injury in the midzonal and pericentral areas. In contrast, in L-arginine-pretreated rat, there were only minor patchy spots of mild necrosis in various areas of liver tissue with dilated sinusoids and dilated central hepatic vein was observed in the liver tissue after $1 \mathrm{~h}$ ischemia followed by $3 \mathrm{~h}$ reperfusion injury. Most areas of the liver tissue L-arginine treated rats exhibited normal structure similar to those of the sham group (Fig.1A).

Normal ultra structural findings was observed in the sham operated controlled group (Fig. 2A). After $1 \mathrm{~h}$ ischemia and $3 \mathrm{~h}$ reperfusion, group II mitochondria of hepatocytes were severe by swollen and had reduction in the number of cristae. Smooth endoplasmic reticulum increased, glycogen granules decreased and nucleus was not well marked and more secondary lysosomes were observed (Fig. 2B). L-Arginine treated on $\mathrm{I} / \mathrm{R}$ induced injury of rat liver showing hepatocytes (Fig. 2C). In this group, hepatocytes severe mitochondrial damages were not displayed and retained the ultra structure of the mitochondria and nucleus.

Cell death of hepatocytes and endothelial cells during reperfusion are characterized by swelling of cells and organelles, release of cell contents, eosinophilia, karyolysis, and induction of inflammation. ${ }^{20)}$ The present histopathology studies of L-arginine treated showed few necrosis, atrophy and hemorrhage at ischemic and reperfusion side and evi-

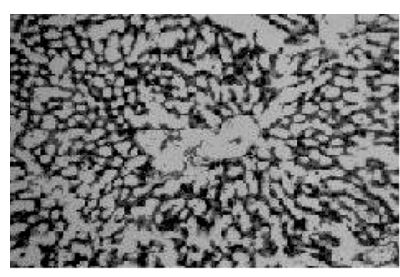

(A)

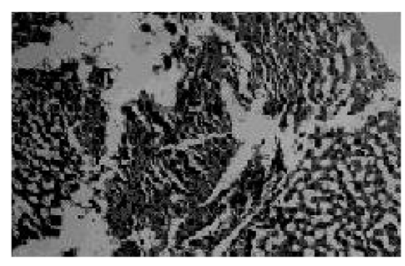

(B)

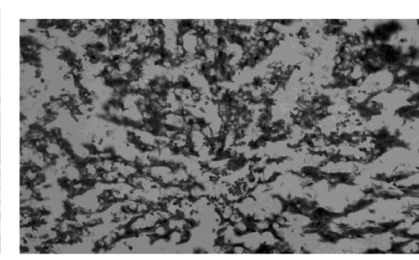

(C)

Fig. 1. Hematoxylin and Eosin (H\&E) Stained Liver Sections Following I/R $(H \& E, \times 400)$

(A) Sham operated liver section shows normal architecture of liver with central vein. (B) I/R liver section shows sinusoidal congestion, cytoplasmic vacuolization, hepatocellular necrosis, and neutrophil infiltration. (C) L-Arginine treated group liver section shows some necrosis, dilated sinusoids, central hepatic vein atrophy and hemorrhage at I/R side and exhibited normal structure similar to those of the sham group. 


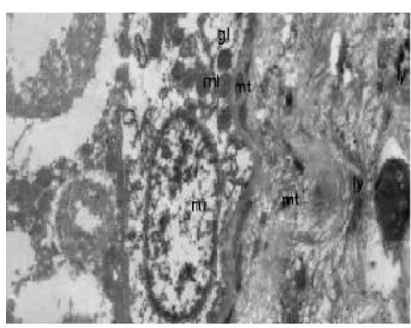

(A)

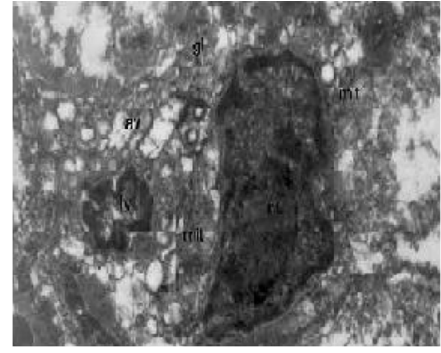

(B)

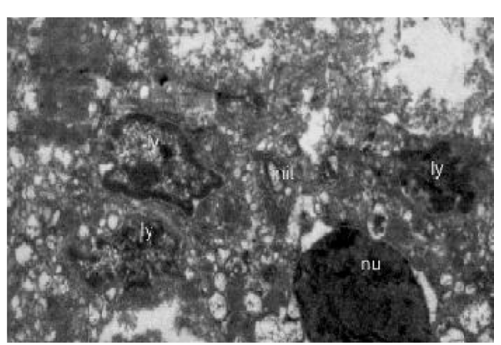

(C)

Fig. 2. Transmission Electron Micrograph Showing the Effect of L-Arginine on I/R Induced Liver Damage

(A) Showing normal morphology of sham operated hepatocytes. (B) Showing severe nucleus and mitochondrial swelling in $\mathrm{I} / \mathrm{R}$ rat. (C) Showing effects of L-arginine on I/R induced hepatocytes where comparable with similar to those of the sham group. mit: Mitochondrion, nu: nucleus, ly: lysosomes, gl: Golgi complex, ly: lysosomes. (Uranyl acetate and lead citrate stain $\times 8000$ )

dently L-arginine protected the severe degenerative changes of $\mathrm{I} / \mathrm{R}$ induced hepatocytes injury.

Lipid peroxidation is related to series of pathologic states, e.g. necrosis, ischemic brain, ${ }^{21)}$ liver, ${ }^{22)}$ and heart disease. ${ }^{23)}$ Lipid peroxidation induces both structural and functional injury to the cell organelle membranes. Reactive oxygen species-induced lipid peroxidation plays important role in the extent of liver damage resulting from I/R. ${ }^{24)}$ In our study studies pretreatment with L-arginine showed substantially attenuated increase in hepatic lipid peroxidation during $\mathrm{I} / \mathrm{R}$ injury.

Thus, the present study provides the evidence that the protective effects of L-arginine against $\mathrm{I} / \mathrm{R}$ injury on rat hepatocyte might be partially due to production of NO and by increased vasodilatation. Further study is needed to establish the molecular mechanism of L-arginine supplementation in $\mathrm{I} / \mathrm{R}$ induced liver injury.

Acknowledgements The authors are grateful to the Dr. R. M. Dubey, Managing Director, IFTM, India and Dr. P. Chaudhury, Principal Scientist, Indian Veterinary Research Institute, India for providing all help and facility to carry out the research work.

\section{REFERENCES}

1) Delva E., Camus Y., Nordlinger B., Ann. Surg., 209, 211-218 (1989).

2) Rudiger H. A., Kang K. J., Sindram D., Riehle H. M., Clavien P. A., Ann. Surg., 235, 400- 407 (2002).

3) Stephenson K. R., Steinberg S. M., Hughes K. S., Ann. Surg., 208, 679-687 (1988).

4) Jaeschke H., Am. J. Physiol. Gastrointest. Liver Physiol., 284, G15G26 (2003)
5) Grace P. A., Br. J. Surg., 81, 637-647 (1994).

6) Glanemann M., Vollmar B., Nussler A. K., Schaefer T., Neuhaus P., Menger M. D., J. Hepatol., 38, 59-66 (2003).

7) Vollmar B., Glasz J., Leiderer R., Post S., Menger M. D., Am. J. Pathol., 145, 1421-1431 (1994).

8) Moncada S. R., Palmar M. J., Higgs E. A., Pharmacol. Rev, 43, 109142 (1991).

9) Kurose I., Kubes P., Suzuki M., Wolf R., Anderson D. C., Paulson J., Miyasaka M., Granger D. N., Circ. Res., 73, 164-171 (1993).

10) Amin A. N., Grrenberg S. S., Than R. R., Fogt F., Loscalzo J., Sadrzadeh S. M., Xie J., Stamler J. S., Gastroenterology, 109, 899907 (1995).

11) Kurose I. R., Wolf M. B., Grisham N., Granger D. N., Circ. Res., 74, 376-382 (1994).

12) Chattopadhyay P., Sharma A. K., Wahi A. K., Ind. J. Gastroenterol., 26, 95-96 (2007).

13) Berry M. N., Friend D. S., J. Cell. Biol., 43, 506-520 (1969).

14) Hibbs J. B., Taintor R. R., Vavrin Z., Rachlin E. M., Biochem. Biophys. Res. Commun., 157, 87-94 (1988).

15) Jordan R. A., Schenkman J. B., Biochem. Pharmacol., 31, 1393-1394 (1982).

16) Ling Y. Q., Shibamoto T., Honda T., Kamikado C., Hironaka E., Hongo M., Koya R. J., Surg. Res., 88, 70-77 (2000).

17) Horie Y. R., Wolf D. C., Anderson P., Granger D. N., J. Clin. Invest., 99, 781-788 (1997).

18) Grace P. A., Br. J. Surg., 81, 637-647 (1994).

19) Jaeschke H., Farhood A., Smith C. W., FASEB J., 4, 3355-3359 (1990).

20) Gujral J. S., Bucci T. J., Farhood A., Jaeschke H., Hepatology, 33, $397-405$ (2001).

21) Bromount C., Marie C., Bralet J., Stroke, 20, 918-924 (1989).

22) Omar R., Nomikos I., Piccorelli G., Savino J., Agrawal N., Gut, 30, 510-514 (1989).

23) Petty M., Grisar J. M., Dow J., Jong W. D., Eur. J. Pharmacol., 179, $241-242(1990)$

24) Atalla S. L., Toledo-Perevra I. H., Mackenzie G. H., Caderna J. P., Transplantation, 40, 584-590 (1985). 